\title{
Combining ordinary and topological finite volume effects for fixed topology simulations
}

\author{
Arthur Dromard*(1), Wolfgang Bietenholz ${ }^{(2)}$, Urs Gerber ${ }^{(2,3)}$, \\ Héctor Mejía-Díaz ${ }^{(2)}$ and Marc Wagner ${ }^{(1)}$ \\ (1) Goethe-Universität Frankfurt am Main \\ Institut für Theoretische Physik \\ Max-von-Laue-Straße 1, D-60438 Frankfurt am Main, Germany \\ (2) Instituto de Ciencias Nucleares \\ Universidad Nacional Autónoma de México \\ A.P. 70-543, C.P. 04510 Distrito Federal, Mexico \\ (3) Instituto de Física y Matemáticas \\ Universidad Michoacana de San Nicolás de Hidalgo \\ Edificio C-3, Apdo. Postal 2-82, C.P. 58040, Morelia, Michoacán, Mexico
}

E-mail: dromardeth.physik.uni-frankfurt.de

\begin{abstract}
In lattice quantum field theories with topological sectors, simulations at fine lattice spacings with typical algorithms - tend to freeze topologically. In such cases, specific topological finite size effects have to be taken into account to obtain physical results, which correspond to infinite volume or unfixed topology. Moreover, when a theory like QCD is simulated in a moderate volume, one also has to overcome ordinary finite volume effects (not related to topology freezing). To extract physical results from simulations affected by both types of finite volume effects, we extend a known relation between hadron masses at fixed and unfixed topology by additionally incorporating ordinary finite volume effects. We present numerical results for SU(2) Yang-Mills theory.
\end{abstract}

The 33rd International Symposium on Lattice Field Theory

14 - 18 July 2015

Kobe International Conference Center, Kobe, Japan*

${ }^{*}$ Speaker. 


\section{Introduction}

In order to extrapolate simulation results in lattice QCD to the continuum limit, the lattice spacing is made as small as possible. However, for an algorithm like Hybrid Monte Carlo, this implies an increase of the topological auto-correlation time, and finally a freezing of the topological charge, for any lattice discretisation [1]. In the case of chirally symmetric fermions, such as overlap fermions, the Monte Carlo history tends to get stuck in one topological sector even for rather large lattice spacing, see e.g. Refs. [2,3]. In specific cases it might be motivated to fix topology on purpose. For example when using a mixed action setup with overlap valence and Wilson sea quarks, one observes an ill-behaved continuum limit due to different near zero-modes in the sea and valence quark sector [4]. Extracting physically meaningful results from the topologically trivial sector could be a solution.

There are several strategies to deal with this problem. The method we are interested in is based on a relation between the physical mass of a hadron and its fixed topology counterpart [57]. This method has already been tested with success in various models [8-17]. So far ordinary finite volume effects have been neglected. However, also these effects can be important for QCD simulations with light pions. Here we describe an extension of the method of Ref. [5], combining both topological and ordinary finite volume effects for $\mathrm{SU}(N)$ Yang-Mills theories, as well as QCD, as anticipated in Ref. [18]. After a brief discussion of ordinary finite volume effects and the method used to extract masses from fixed topology, we present the extended equations and the results of a numerical test in SU(2) Yang-Mills theory.

\section{Ordinary finite volume effects}

The difficulties in lattice simulations related to finite volume effects are well-known. A finite volume with periodic boundary conditions allows the particle to interact with its own copies. This artificial interaction causes a shift in the value of the mass obtained by simulations.

This mass shift has been rigorously analysed in Ref. [19]. Different results were obtained for $\mathrm{SU}(N)$ gauge theory and for QCD, due to the different masses of the lightest stable particle, namely the $0^{++}$glueball and the pion. For Yang-Mills theories one obtains

$$
M(L)-M(L=\infty) \propto \frac{1}{L} \exp \left(-\frac{\sqrt{3} m_{\mathrm{g}} L}{2}\right),
$$

where $m_{\mathrm{g}}$ is the mass of the lightest glueball, $\left(J^{P C}=0^{++}\right)$and $L$ is the spatial extent of the lattice. The corresponding QCD relation reads

$$
m_{\pi}(L)-m_{\pi}(L=\infty) \propto \frac{1}{L} K_{1}\left(m_{\pi} L\right),
$$

where $m_{\pi}$ is the pion mass and $K_{1}$ the modified Bessel function. We confronted eq. (2.1) with lattice SU(2) Yang-Mills results for the static quark-antiquark potential $\hat{\mathscr{V}}_{q \bar{q}}$ (hats denote quantities in lattice units). For this purpose, we computed $\hat{\mathscr{V}}_{q \bar{q}}(\hat{r}=3)$ in the volumes $\hat{V}=\hat{L}^{4}, \hat{L}=11,12, \ldots, 16$ and 18 , based on 4000 configurations in each volume. ${ }^{1}$ The results are in excellent agreement with

\footnotetext{
${ }^{1}$ The same set of configurations was used for the results reported in Section 3 to 5. Identifying the Sommer parameter $r_{0}$ with $0.46 \mathrm{fm}$ one obtains a lattice spacing of $a \approx 0.073 \mathrm{fm}$.
} 
the theory. For $\hat{L} \geq 14$ the finite volume effects are negligible, but in smaller volumes they have to be taken into account. From the fit of eq. (2.1) to the lattice results we can extract the glueball mass to a good precision, $\hat{m}_{\mathrm{g}}=0.74(4)$ (cf. Figure 2 in [18]) This is in excellent agreement with the result obtained by a standard lattice computation of a glueball 2-point correlation function, $\hat{m}_{\mathrm{g}}=0.723(23)[20]$.

\section{Topological finite volume effects}

Fixing the topology, i.e. the restriction to only one topological sector, entails additional typically even stronger finite volume effects. A relation between a physical hadron mass $M$ and the hadron mass $M_{Q, V}$ obtained at fixed topological charge $Q$, in a finite space-time volume $V$, has been derived in Ref. [5],

$$
M_{Q, V}=M+\frac{1}{2 \chi_{t} V} M^{(2)}\left(1-\frac{Q^{2}}{\chi_{t} V}\right)+\mathscr{O}\left(\frac{1}{\left(\chi_{t} V\right)^{2}}\right),
$$

where $M^{(2)}$ is the second derivative of $M$ with respect to the vacuum angle $\theta$, at $\theta=0$, and $\chi_{t}$ is the topological susceptibility. One can see that fixed topology causes finite volume effects, which are only suppressed by powers of the inverse volume.

In practice, the physical mass $M$ can be extracted by a fit of eq. (3.1) to numerical results for $M_{Q, V}$ in various volumes and topological sectors. This method has been tested in Refs. [7$18]$ with success. The static potential $\hat{\mathscr{V}}_{q \bar{q}, Q, V}$ has been computed for different separations $(\hat{r}=$ $1 \ldots 6)$ in different topological sectors, $|Q|=0,1,2$, and various volumes, $\hat{V}=14^{4}, 15^{4}, 16^{4}, 18^{4}$. Figure 1 shows $\hat{\mathscr{V}}_{q \bar{q}, Q, V}(\hat{r}=3)$; there is a clear distinction between topological sectors, especially in small volumes. This calls for an indirect method to extract physical results. The curves represent a global fit of eq. (3.1) to the numerical results, which is of good quality, $\chi^{2} /$ d.o.f. $\lesssim 1$. The extrapolated potential $\hat{\mathscr{V}}_{q \bar{q}}(\hat{r}=3)=0.1646(2)$ is in excellent agreement with the corresponding straight computation at unfixed topology, $\hat{\mathscr{V}}_{q \bar{q}}(\hat{r}=3)=0.16455(7)$.

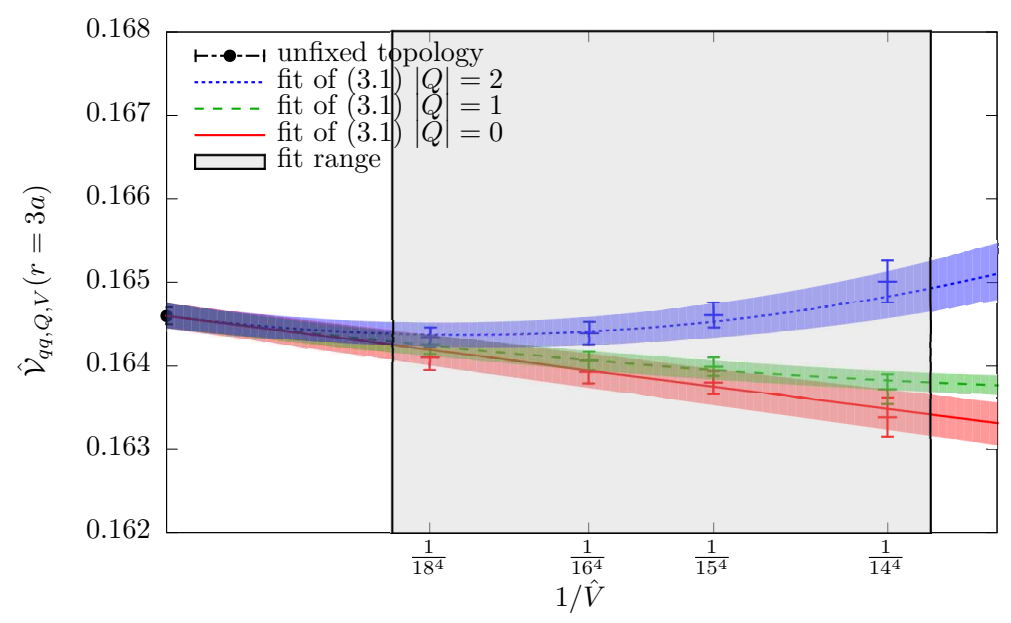

Figure 1: The static potential (in lattice units), $\hat{\mathscr{V}}_{q \bar{q}, Q, V}(\hat{r}=3)$, as a function of the inverse lattice volume, $1 / \hat{V}$. The curves represent the fit of eq. (3.1) to the lattice results in the volumes $\hat{V}=14^{4}, 15^{4}, 16^{4}, 18^{4}$.

In $\sigma$-model studies, also $\hat{\chi}_{t}$ could be evaluated well in this manner $[13,16]$. In $4 \mathrm{~d}$ Yang-Mills theory, however, the determination of $\hat{\chi}_{t}$ turned out to be plagued by large statistical errors. A 
more promising method for that purpose has been suggested in Ref. [6], which showed that the correlation of the topological charge density $q(x)$ at fixed topology behaves as

$$
\langle q(x) q(0)\rangle_{Q, V} \underset{|x| \rightarrow \infty}{\approx}-\frac{\chi_{t}}{V}\left(1-\frac{Q^{2}}{\chi_{t} V}\right)+\mathscr{O}\left(\frac{1}{V^{2}}\right) .
$$

Eq. (3.2) implies that - at a large separation $|x|$ - the correlation $\langle q(x) q(0)\rangle_{Q, V}$ should reach a plateau, and that its value determines $\chi_{t}$. The advantage of this method is the need of only one volume and one topological sector.

Figure 2 has been generated in the volume $\hat{V}=16^{4}$, based on an all-to-all computation of the correlator. This was carried out after performing 8 cooling sweeps, in order to smooth out the ultra-violet fluctuations without destroying the topological structure. Each fit of the plateau works with $\chi^{2} /$ d.o.f. $\approx 1$. This suggests that this method is promising indeed.

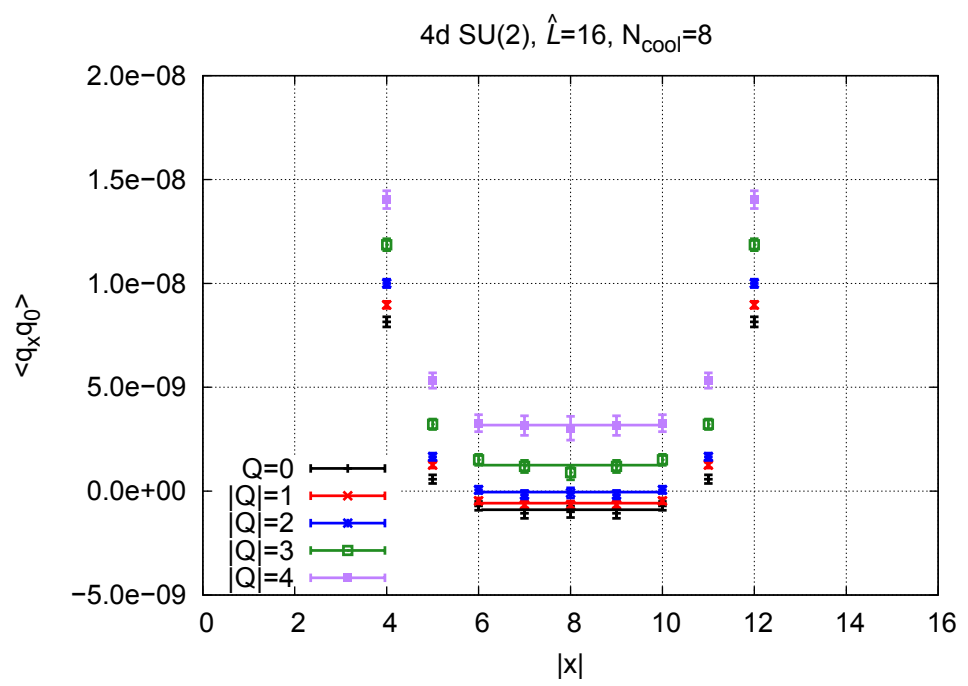

Figure 2: The correlation $\left\langle q_{x} q_{0}\right\rangle_{|Q|}$ as a function of the on-axis separation $|x|$, after 8 cooling sweeps, for the lattice volume $\hat{V}=16^{4}$.

\section{Combining topological and ordinary finite volume effects}

The results in Section 3 were obtained for volumes where ordinary finite volume effects are negligible. This was rather easy, as $\mathrm{SU}(2)$ theory is a special case since even the lightest glueball has a considerable mass, which implies strongly suppressed ordinary finite volume effects. As indicated in Section 2, and applied in Section 3, one has to discard volumes with $\hat{L}<14$, i.e. box sizes $L \lesssim 1 \mathrm{fm}$. On the other hand, in QCD the lightest particle is the pion, which is much lighter than any glueball in $\mathrm{SU}(2)$ Yang-Mills theory. Therefore one expects persistent ordinary finite volume effects up to larger lattices.

In order to understand how eq. (3.1) is affected by ordinary finite volume effects, we also computed the static $q \bar{q}$-potential for smaller lattices, $\hat{L}<14$, which are affected by significant finite volume effects. In Figure 3 we show results for seven volumes in the range $11^{4} \ldots 18^{4}$, for $|Q|=0,1$ and 2. The lines represent the fit of the formula (3.1) in volumes $\hat{V}>13^{4}$. For these volumes, this equation is well compatible with our data. For smaller volumes, however, the discrepancies are significant and clearly show that ordinary finite volume effects have to be taken 


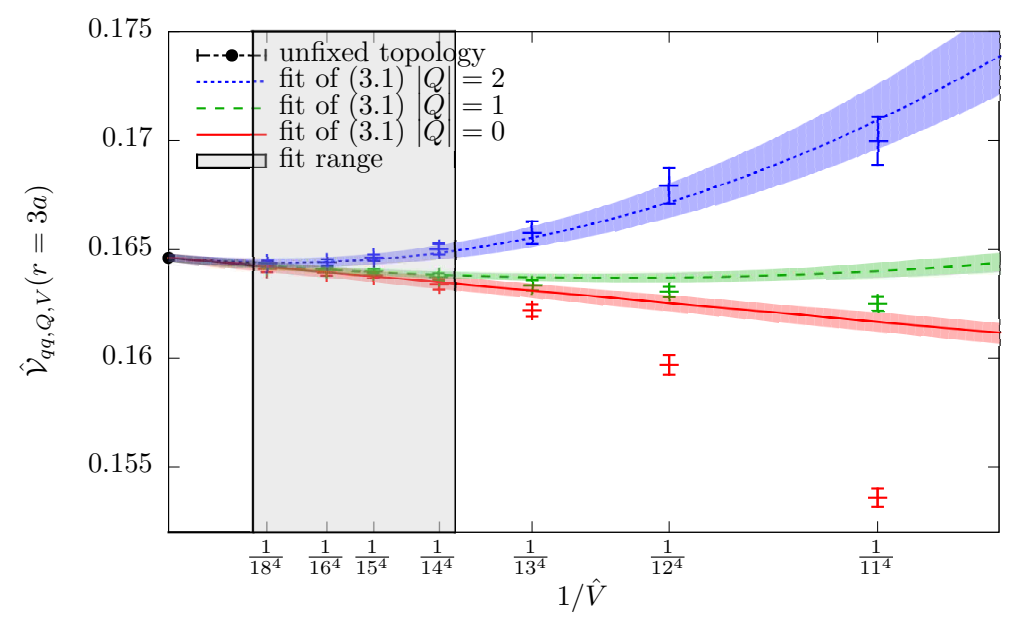

Figure 3: $\hat{\mathscr{V}}_{q \bar{q}, Q, V}(\hat{r}=3)$ as a function of $1 / \hat{V}$. The curves represent the fit of eq. (3.1) to the lattice results in the four largest volumes, $\hat{V}=14^{4}, 15^{4}, 16^{4}, 18^{4}$. There is are sizable discrepancies between these curves and the lattice data in the smaller volumes, $\hat{V}=11^{4}, 12^{4}, 13^{4}$.

into account. Therefore it is highly desirable to combine eq. (3.1) with eq. (2.1) or (2.2), in order to obtain a formula which captures both ordinary and topological finite size effects. Comparing the topological sectors, one notices that the difference between the curve and the data is larger for topological charge $Q=0$ than for $|Q|=1,2$. For the latter the finite volume effects seem negligible. This different behaviour underlines the topological charge dependence of ordinary finite volume effects at fixed topology.

To derive such expressions, one first has to compute ordinary finite volume effects also at non-vanishing $\theta$ angles, using equations analogous to (2.1) or (2.2). To this end, we employed the Lüscher method [19], which is universal and therefore applicable to $\mathrm{SU}(N)$ Yang-Mills theory. Starting from the finite volume equation at fixed $\theta$, one has to perform a calculus similar the one leading to eq. (3.1), see Ref. [7]. In Yang-Mills theory, the expression, which captures both types of finite volume effects, is

$$
\begin{aligned}
M_{Q, L} & \approx M+\frac{1}{2 \chi_{t} V} M^{(2)}\left(1-\frac{Q^{2}}{\chi_{t} V}\right)-\frac{A}{L} \exp \left(-\frac{\sqrt{3} m_{\mathrm{g}} L}{2}\right) \\
& +\frac{1}{2 \chi_{t} V}\left(B-A \sqrt{3} m_{\mathrm{g}}^{(2)}\right)\left(1-\frac{Q^{2}}{\chi_{t} V}\right) \exp \left(-\frac{\sqrt{3} m_{\mathrm{g}} L}{2}\right),
\end{aligned}
$$

where $M_{Q, L}$ is a hadron mass in a finite volume in one topological sector, whereas $M$ and $m_{\mathrm{g}}$ are the physical mass (at infinite volume and $\theta=0$ ) of the hadron and the $0^{++}$glueball. $A$ and $B$ are coefficients, which are independent of $V$ and $Q$, and (2) denotes the second derivative with respect to $\theta$ at $\theta=0$. The corresponding formula for the pion mass in QCD reads

$$
\begin{aligned}
m_{\pi, Q, L} & \approx m_{\pi}+\frac{m_{\pi}^{(2)}}{2 \chi_{t} V}\left(1-\frac{Q^{2}}{\chi_{t} V}\right)+\frac{3}{16 \pi^{2}} \frac{m_{\pi}^{2}}{F_{\pi}^{2}} \frac{K_{1}(x)}{x}+\frac{m_{\pi}^{(2)} E}{2 \chi_{t} V}\left(1-\frac{2 Q^{2}}{\chi_{t} V}\right) \frac{K_{1}(x)}{x} \\
& +\frac{3}{32 \pi^{2} \chi_{t} V} \frac{m_{\pi}^{2}}{F_{\pi}^{2}}\left(1-\frac{Q^{2}}{\chi_{t} V}\right)\left[\left(\frac{m_{\pi}^{(2)}}{m_{\pi}}-2 \frac{F_{\pi}^{(2)}}{F_{\pi}}\right) \frac{K_{1}(x)}{x}-\frac{1}{2} \frac{m_{\pi}^{(2)}}{m_{\pi}}\left(K_{0}(x)+K_{2}(x)\right)\right],
\end{aligned}
$$

where $F_{\pi}$ is the pion decay constant, $E$ is another constant (independent of $V$ and $Q$ ), $K_{n}$ is the modified Bessel function and $x=m_{\pi} L$. 


\begin{tabular}{|c|c|c|c|}
\hline & $\hat{\mathscr{V}}_{q q}(\hat{r}=3)$ & $\hat{m}_{\mathrm{g}}$ & $\hat{\chi}_{t} \times 10^{5}$ \\
\hline \hline fit based on eq. (4.1) & $0.16437(15)$ & $0.67(10)$ & $9.5(2.0)$ \\
\hline result at unfixed topology [20,21] & $0.16455(7)$ & $0.723(23)$ & $7.0(0.9)$ \\
\hline
\end{tabular}

Table 1: Results for the static potential $\hat{\mathscr{V}}_{q \bar{q}}(\hat{r}=3)$, the mass $\hat{m}_{\mathrm{g}}$ of the $J^{P C}=0^{++}$glueball and the topological susceptibility $\hat{\chi}_{t}$, obtained by a fit of eq. (4.1) to fixed topology lattice results for $\hat{\mathscr{V}}_{q \bar{q}, Q, V}(\hat{r}=3)$.

\section{Numerical test in $\mathrm{SU}(2)$ gauge theory}

Figure 4 shows the same data points as Figure 3, but now with a fit according to eq. (4.1), which includes the ordinary finite volume effects. The fitting parameters are $\hat{M}, \hat{M}^{(2)}, \hat{m}_{\mathrm{g}}, \hat{m}_{\mathrm{g}}^{(2)}, A, B$ and $\hat{\chi}_{t}$. We see that eq. (4.1) matches the data very well, with $\chi^{2} /$ d.o.f. $<1$; the explicit results are given in Table 1. They all agree with the literature, albeit with considerable errors for $\hat{m}_{\mathrm{g}}$ and $\hat{\chi}_{t}$. In particular, for the topological susceptibility the error is larger than $20 \%$, hence for that quantity a different approach should be used, such as the method discussed in Section 3. Nevertheless the evaluation of $\hat{\mathscr{V}}_{q \bar{q}}$ is achieved to a high precision, with an error below $0.1 \%$.

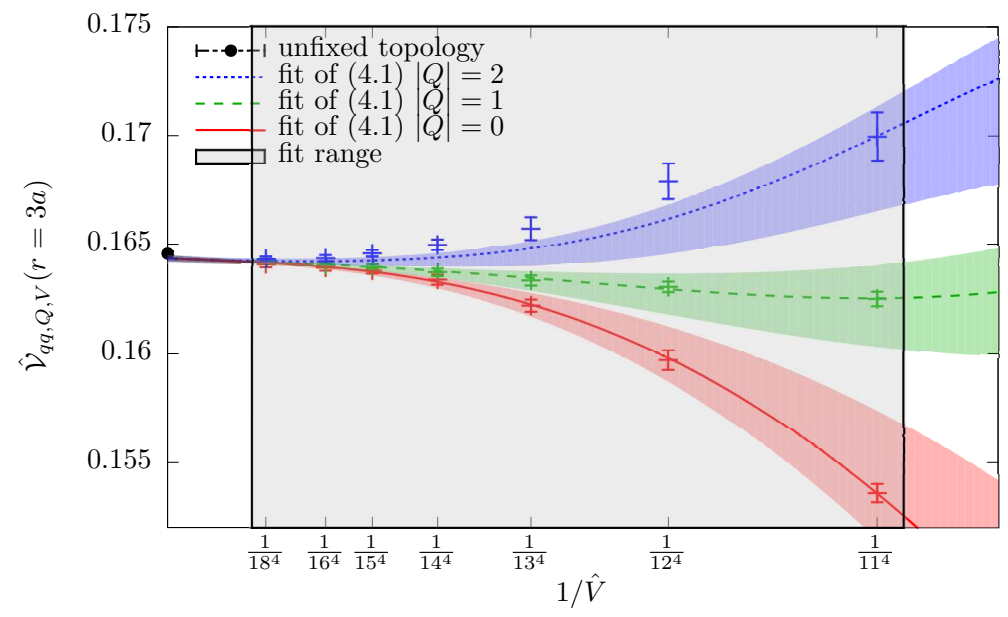

Figure 4: $\hat{\mathscr{V}}_{q \bar{q}, Q, V}(\hat{r}=3)$ as a function of $1 / \hat{V}$. The curves represent the fit of eq. (4.1) to the lattice results in all volumes $\hat{V}=11^{4}, \ldots, 18^{4}$. We obtain very good agreement, even at small volumes and $Q=0$.

To conclude, we have included ordinary finite volume effects in a relation between a hadron mass at fixed topology and finite volume, and the corresponding physical mass. We successfully tested this new relation in SU(2) Yang-Mills theory for the static quark-antiquark potential. Due to this extension, the method becomes more promising in QCD applications. This is currently under investigation.

\section{Acknowledgements}

A.D. and M.W. acknowledge support by the Emmy Noether Programme of the DFG (German Research Foundation), grant WA 3000/1-1. W.B., U.G. and H.M.-D. acknowledge support by the Consejo Nacional de Ciencia y Tecnología (CONACYT) through projects CB-2010/155905 and CB-2013/222812, as well as DGAPA-UNAM, grant IN107915. 
This work was supported in part by the Helmholtz International Center for FAIR within the framework of the LOEWE program launched by the State of Hesse. Calculations on the LOEWECSC high-performance computer of Johann Wolfgang Goethe-University Frankfurt am Main were conducted for this research. We would like to thank HPC-Hessen, funded by the State Ministry of Higher Education, Research and the Arts, for programming advice.

\section{References}

[1] M. Lüscher and S. Schaefer, JHEP 1107, 036 (2011) [arXiv:1105.4749 [hep-lat]].

[2] S. Aoki et al. [JLQCD Collaboration], Phys. Rev. D 78, 014508 (2008) [arXiv:0803.3197 [hep-lat]].

[3] Sz. Borsanyi, Z. Fodor, S.D. Katz, S. Krieg, T. Lippert, D. Nogradi, F. Pittler, K.K. Szabo and B.C. Toth, arXiv: 1510.03376 [hep-lat].

[4] K. Cichy, G. Herdoiza and K. Jansen, Nucl. Phys. B 847, 179 (2011) [arXiv:1012.4412 [hep-lat]].

[5] R. Brower, S. Chandrasekharan, J. W. Negele and U.-J. Wiese, Phys. Lett. B 560, 64 (2003) [hep-lat/0302005].

[6] S. Aoki, H. Fukaya, S. Hashimoto and T. Onogi, Phys. Rev. D 76, 054508 (2007) [arXiv:0707.0396 [hep-lat]].

[7] A. Dromard and M. Wagner, Phys. Rev. D 90, 074505 (2014) [arXiv:1404.0247 [hep-lat]].

[8] W. Bietenholz, I. Hip, S. Shcheredin and J. Volkholz, Eur. Phys. J. C 72, 1938 (2012) [arXiv:1109.2649 [hep-lat]].

[9] W. Bietenholz and I. Hip, PoS LATTICE 2008, 079 (2008) [arXiv:0808.3049 [hep-lat]].

[10] W. Bietenholz and I. Hip, J. Phys. Conf. Ser. 378, 012041 (2012) [arXiv:1201.6335 [hep-lat]].

[11] A. Dromard and M. Wagner, PoS LATTICE 2013, 339 (2014) [arXiv:1309.2483 [hep-lat]].

[12] C. Czaban and M. Wagner, PoS LATTICE 2013, 465 (2013) [arXiv:1310.5258 [hep-lat]].

[13] I. Bautista et al., arXiv:1402.2668 [hep-lat].

[14] C. Czaban, A. Dromard and M. Wagner, Acta Phys. Polon. Supp. 7, 551 (2014) [arXiv:1404.3597 [hep-lat]].

[15] A. Dromard, C. Czaban and M. Wagner, PoS LATTICE 2014, 321 (2014) [arXiv:1410.4333 [hep-lat]].

[16] U. Gerber et al., PoS LATTICE 2014 (2014) [arXiv:1410.0426 [hep-lat]].

[17] I. Bautista et al., arXiv:1503.06853 [hep-lat].

[18] A. Dromard, W. Bietenholz, U. Gerber, H. Mejía-Díaz and M. Wagner, Acta Phys. Polon. Supp. 8, no. 2, 391 (2015) [arXiv:1505.03435 [hep-lat]].

[19] M. Lüscher, Commun. Math. Phys. 104, 177 (1986).

[20] M. J. Teper, hep-th/9812187.

[21] P. de Forcrand, M. García Pérez and I. O. Stamatescu, Nucl. Phys. B 499, 409 (1997) [hep-lat/9701012]. 